\title{
Existence Theorems for Solvability of a Functional Equation Arising in Dynamic Programming
}

\author{
Deepmala \\ School of Studies in Mathematics, Pt. Ravishankar Shukla University, Raipur (C.G.) 492010, India \\ Correspondence should be addressed to Deepmala; dmrai23@gmail.com \\ Received 4 January 2014; Revised 5 March 2014; Accepted 5 March 2014; Published 8 April 2014 \\ Academic Editor: Nawab Hussain
}

Copyright ( 2014 Deepmala. This is an open access article distributed under the Creative Commons Attribution License, which permits unrestricted use, distribution, and reproduction in any medium, provided the original work is properly cited.

The paper deals with the existence, uniqueness, and iterative approximations of solutions for the functional equations arising in dynamic programming of multistage decision making processes in Banach spaces $B C(S)$ and $B(S)$ and complete metric space $B B(S)$, respectively. Our main results extend, improve, and generalize the results due to several authors. Some examples are also given to demonstrate the advantage of our results over existing one in the literature.

\section{Introduction}

In this paper, we introduce and study the existence and uniqueness of solutions for the following functional equation arising in dynamic programming of multistage decision processes:

$$
\begin{array}{r}
f(x)=\operatorname{opt}_{y \in D}\left\{u(x, y)+\operatorname{opt}\left\{v(x, y), p_{i}(x, y)+q_{i}(x, y)\right.\right. \\
\left.\left.\times f\left(a_{i}(x, y)\right): i=1,2,3\right\}\right\}, \\
\forall x \in S,
\end{array}
$$

where "opt" denotes the "sup" or "inf," $x$ and $y$ stand for the state and decision vectors, respectively, $a_{i}$ represents the transformation of the processes, and $f(x)$ denotes the optimal return function with initial state $x$.

It is clear that (1) includes many functional equations and system of functional equations as special case, respectively. Bellman [1] was the first to investigate the existence and uniqueness of solutions for the following functional equation:

$$
\begin{gathered}
f(x)=\inf _{y \in D} \max \{r(x, y), s(x, y), f(b(x, y))\}, \quad x \in S, \\
f(x)=\inf _{y \in D} \max \{r(x, y), f(b(x, y))\}, \quad x \in S
\end{gathered}
$$

Bhakta and Mitra [2] obtained the existence and uniqueness of solutions for the functional equations

$$
f(x)=\sup _{y \in D}\{p(x, y)+A(x, y, f(a(x, y)))\}, \quad x \in S
$$

in a Banach space $B(S)$ and

$$
f(x)=\sup _{y \in D}\{p(x, y)+f(a(x, y))\}, \quad x \in S
$$

in $B B(S)$, respectively. Bhakta and Choudhury [3] established the existence of solutions for the functional equations (2) in $B B(S)$.

In 2003, Liu and Ume [4] pointed out that the form of the functional equations of dynamic programming is as follows:

$$
\begin{array}{r}
f(x)=\operatorname{opt}_{y \in D}\{a[u(x, y)+f(T(x, y))] \\
+b \operatorname{opt}[v(x, y), f(T(x, y))]\}, \\
x \in S, \quad a+b=1 .
\end{array}
$$

In 2004, Liu et al. [5] obtained an existence, uniqueness, and iterative approximation of solutions for the functional equation

$$
f(x)=\underset{y \in D}{\operatorname{opt}}\left\{u ( x , y ) + \text { opt } \left\{p_{i}(x, y)+q_{i}(x, y)\right.\right.
$$

$$
\left.\left.\times f_{i}\left(a_{i}(x, y)\right): i=1,2\right\}\right\},
$$


In 2006, Liu et al. [6] provided the sufficient conditions which ensure the existence and uniqueness and iterative approximation of solution for the functional equation

$$
\begin{gathered}
f(x)=\underset{y \in D}{\operatorname{opt}}\{p(x, y)+q(x, y) f(a(x, y)) \\
+ \text { opt }\{r(x, y), s(x, y) f(b(x, y)), \\
t(x, y) f(c(x, y))\}\}, \\
x \in S .
\end{gathered}
$$

In 2007, Liu and Kang [7] studied the following functional equation:

$$
\begin{aligned}
f(x)=\inf _{y \in D} \max & \{p(x, y), f(a(x, y)), q(x, y) \\
& +f(b(x, y))\}, \quad \forall x \in S
\end{aligned}
$$

and gave an existence and uniqueness result of solution for the functional equation.

In 2011, Jiang et al. [8] investigated the following functional equation:

$$
\begin{array}{r}
f(x)=\underset{y \in D}{\operatorname{opt} \text { opt }}\{p(x, y), q(x, y) f(a(x, y)), r(x, y) \\
\times f(b(x, y)), s(x, y) f(c(x, y))\}, \\
\forall x \in S
\end{array}
$$

and gave some existence and uniqueness results and iterative approximations of solutions for the functional equation in $B B(S)$.

In Section 2, we recall some basic concepts, notations, and lemmas. In Section 3, we utilize the fixed point theorem due to Boyd and Wong [9] to establish the existence, uniqueness, and iterative approximation of solution for the functional equation (1) in Banach spaces and complete metric spaces. Also, we construct some nontrivial examples to explain our results. The results presented here generalize, improve, and extend the results of Bellman [1], Bhakta and Mitra [2], Bhakta and Choudhury [3], Liu and Ume [4], Liu et al. [5], Liu et al. [6], Liu and Kang [7], Jiang et al. [8], Pathak and Deepmala [10], and Liu [11]. The existence problems of solutions of various functional equations arising in dynamic programming are both theoretical and practical interest. Fixed point theorems are applied in various fields (see [12-16]). Here, we use a fixed point theorem to show the solvability of the functional equation arising in dynamic programming.

\section{Preliminaries}

Throughout this paper, we assume that $\mathbb{R}=(-\infty,+\infty), R^{+}=$ $[0,+\infty)$, and $R^{-}=(-\infty, 0]$. For any $t \in \mathbb{R},[t]$ denotes the largest integer not exceeding $t$ and $(X,\|\cdot\|)$ and $\left(Y,\|\cdot\|^{\prime}\right)$ are real Banach spaces. Let $S \subseteq X$ be the state space and let $D \subseteq Y$ be decision space. Define

$$
\begin{aligned}
& \Phi_{1}=\left\{\varphi: \varphi: R^{+} \rightarrow R^{+} \text {is nondecreasing }\right\} \\
& \Phi_{2}=\left\{(\varphi, \psi): \varphi, \psi \in \Phi_{1}, \psi(t)>0\right. \text { and } \\
& \left.\sum_{n=0}^{\infty} \psi\left(\varphi^{n}(t)\right)<\infty \text { for } t>0\right\}, \\
& \Phi_{3}=\left\{(\varphi, \psi): \varphi, \psi \in \Phi_{1}, \psi(t)>0\right. \text { and } \\
& \left.\lim _{n \rightarrow \infty} \psi\left(\varphi^{n}(t)\right)=0 \text { for } t>0\right\}, \\
& \Phi_{4}=\left\{\varphi: \varphi \in \Phi_{1} \text { and } \sum_{n=0}^{\infty} \varphi^{n}(t)<\infty \text { for } t>0\right\}, \\
& B(S)=\{f: f: S \rightarrow \mathbb{R} \text { is bounded }\}, \\
& B C(S)=\{f: f \in B(S) \text { is continuous }\}, \\
& B B(S)=\{f: f: S \rightarrow \mathbb{R} \text { is bounded on bounded } \\
& \text { subsets of } S\} .
\end{aligned}
$$

Here, $\left(B(S),\|\cdot\|_{1}\right)$ and $\left(B C(S),\|\cdot\|_{1}\right)$ are Banach spaces with norm $\|f\|_{1}=\sup _{x \in S}|f(x)|$. For any positive integer $k$ and $f$, $g \in B B(S)$, let

$$
\begin{aligned}
d_{k}(f, g) & =\sup \{|f(x)-g(x)|: x \in \bar{B}(0, k)\} \\
d(f, g) & =\sum_{k=1}^{\infty} \frac{1}{2^{k}} \cdot \frac{d_{k}(f, g)}{1+d_{k}(f, g)},
\end{aligned}
$$

where $\bar{B}(0, k)=\{x: x \in S$ and $\|x\| \leq k\}$. Then, $\left\{d_{k}\right\}_{k \geq 1}$ is a countable family of pseudometrices on $B B(S)$. A sequence $\left\{x_{n}\right\}_{n \geq 1}$ in $B B(S)$ is said to converge to a point $x \in B B(S)$ if for any $k \geq 1, d_{k}\left(x_{n}, x_{m}\right) \rightarrow 0$ as $n, m \rightarrow \infty$. It is clear that $(B B(S), d)$ is a complete metric space. A metric space $(M, \rho)$ is said to metrically convex if for each $x, y \in M$, there is a $z \neq x, y$ for which $\rho(x, y)=\rho(x, z)+\rho(z, y)$. Clearly, any Banach space is metrically convex.

Theorem 1 (see [9]). Suppose that $(M, \rho)$ is a complete metrically convex metric space and that $f: M \rightarrow M$ satisfies

$$
\rho(f(x), f(y)) \leq \varphi(\rho(x, y)) \quad \text { for } x, y \in M
$$

where $\varphi: \bar{P} \rightarrow R^{+}$satisfies $\varphi(t)<t$ for $t \in \bar{P}-\{0\}$, where $P=\{\rho(x, y): x, y \in M\}$ and $\bar{P}$ denotes the closure of $P$. Then, $f$ has a fixed point $u \in M$ and $\lim _{n \rightarrow \infty} f^{n}(x)=u$, for each $x \in M$.

Lemma 2 (see $[8]) .\left\{a_{i}, b_{i}: 1 \leq i \leq n\right\} \subseteq \mathbb{R}$. Then,

$$
\begin{aligned}
& \operatorname{|opt}\left\{a_{i}: 1 \leq i \leq n\right\}-\text { opt }\left\{b_{i}: 1 \leq i \leq n\right\} \mid \\
& \quad \leq \max \left\{\left|a_{i}-b_{i}\right|: 1 \leq i \leq n\right\} .
\end{aligned}
$$

Lemma 3 (see [8]). (i) Assume that $A: S \times D \rightarrow \mathbb{R}$ is a mapping such that opt ${ }_{y \in D} A\left(x_{0}, y\right)$ is bounded for some $x_{0} \in S$. Then,

$$
\left|\operatorname{opt}_{y \in D} A\left(x_{0}, y\right)\right| \leq \sup _{y \in D}\left|A\left(x_{0}, y\right)\right| \text {. }
$$


(ii) Assume that $A, B: S \times D \rightarrow \mathbb{R}$ is a mapping such that opt ${ }_{y \in D} A\left(x_{1}, y\right)$ and opt $_{y \in D} B\left(x_{2}, y\right)$ is bounded for some $x_{1}, x_{2} \in S$. Then,

$$
\begin{aligned}
& \left|\operatorname{opt}_{y \in D} A\left(x_{1}, y\right)-\underset{y \in D}{\operatorname{opt}} B\left(x_{2}, y\right)\right| \\
& \quad \leq \sup _{y \in D}\left|A\left(x_{1}, y\right)-B\left(x_{2}, y\right)\right| .
\end{aligned}
$$

\section{Main Results}

In this section, we discuss existence and uniqueness of solutions in $B C(S)$ and $B(S)$.

Theorem 4. Let $S$ be compact. Let $u, v, p_{i}, q_{i}: S \times D \rightarrow \mathbb{R}$ and $a_{i}: S \times D \rightarrow S$ for $i=1,2,3$ and satisfy the following conditions:

$\left(C_{1}\right) u, v$ and $p_{i}$ are bounded for $i=1,2,3$.

$\left(C_{2}\right)$ for each $x_{0} \in S, u(x, y) \rightarrow u\left(x_{0}, y\right), v(x, y) \rightarrow$ $v\left(x_{0}, y\right), p_{i}(x, y) \rightarrow p_{i}\left(x_{0}, y\right), q_{i}(x, y) \rightarrow$ $q_{i}\left(x_{0}, y\right), a_{i}(x, y) \rightarrow a_{i}\left(x_{0}, y\right)$ as $x \rightarrow x_{0}$ uniformly for $y \in D$ and $i=1,2,3$.

$\left(C_{3}\right) \max \left\{\left|q_{i}(x, y)\right|: i=1,2,3\right\} \leq \alpha$, for some $\alpha \in(0,1)$ and $(x, y) \in S \times D$.

Then, the functional equation (1) possesses a unique solution $w \in B C(S)$ and $\left\{H^{n} h\right\}_{n \geq 1}$ converges to $w$ for each $h \in B C(S)$, where $H$ is defined by

$$
\begin{aligned}
H h(x)=\operatorname{opt}_{y \in D}\{u(x, y) & \\
+ \text { opt }\{ & \begin{aligned}
& \\
& \\
& \left.\left.\left.\times h(x, y), p_{i}(x, y)\right): i=1,2,3\right\}\right\}, \\
& q_{i}(x, y) \\
& \forall x \in S .
\end{aligned}
\end{aligned}
$$

Proof. Let $h \in B C(S)$ and $x_{0} \in S$ and $\epsilon>0$; it follows from $\left(C_{1}\right),\left(C_{2}\right)$ and compactness of $S$ that there exists a constant $M>0, \delta>0$, and $\delta_{1}>0$ such that

$$
\begin{array}{r}
\max \left\{|u(x, y)|,|v(x, y)|,\left|p_{i}(x, y)\right|: i=1,2,3\right\} \leq M, \\
\forall(x, y) \in S \times D,
\end{array}
$$

$\max \left\{|h(x)|,\left|h\left(a_{i}(x, y)\right)\right|: i=1,2,3\right\} \leq M$,

$$
\forall(x, y) \in S \times D,
$$

$\sup _{y \in D}\left\{\left|u(x, y)-u\left(x_{0}, y\right)\right|\right\} \leq \frac{\epsilon}{4}, \quad$ with $\left\|x-x_{0}\right\|<\delta$,

$$
\begin{array}{r}
\max \left\{\left|p_{i}(x, y)-p_{i}\left(x_{0}, y\right)\right|: i=1,2,3\right\} \leq \frac{\epsilon}{4}, \\
\text { with }\left\|x-x_{0}\right\|<\delta, \\
\max \left\{\left|q_{i}(x, y)-q_{i}\left(x_{0}, y\right)\right|: i=1,2,3\right\} \leq \frac{\epsilon}{4 M}, \\
\text { with }\left\|x-x_{0}\right\|<\delta, \\
\left|h\left(x_{1}\right)-h\left(x_{2}\right)\right|<\frac{\epsilon}{4}, \quad \forall x_{1}, x_{2} \in S, \\
\text { with }\left\|x_{1}-x_{2}\right\|<\delta_{1}, \\
\max \left\{\left\|a_{i}(x, y)-a_{i}\left(x_{0}, y\right)\right\|: i=1,2,3\right\}<\delta_{1}, \\
\forall(x, y) \in S \times D \quad \text { with }\left\|x-x_{0}\right\|<\delta,
\end{array}
$$

$$
\begin{aligned}
& |H h(x)| \\
& \begin{array}{l}
\leq \sup _{y \in D} \mid\left\{u(x, y)+\operatorname{opt}\left\{v(x, y), p_{i}(x, y)\right.\right. \\
+q_{i}(x, y) h\left(a_{i}(x, y)\right) \\
\quad: i=1,2,3\}\} \mid
\end{array} \\
& \begin{array}{l}
\leq \sup _{y \in D}\left\{|u(x, y)| \quad \times \max \left\{|v(x, y)|,\left|p_{i}(x, y)\right|+\left|q_{i}(x, y)\right|\right.\right. \\
\left.\left.\quad \times\left|h\left(a_{i}(x, y)\right)\right|: i=1,2,3\right\}\right\}
\end{array} \\
& \leq M+\max \{M, M+\alpha M\} \\
& \leq 2 M+\alpha M .
\end{aligned}
$$

Thus, $H$ is bounded.

In light of $\left(C_{3}\right),(15),(17)$, and Lemmas 2 and 3, we deduce that for all $(x, y) \in S \times D$ with $\left\|x-x_{0}\right\|<\delta$

$$
\begin{aligned}
& \left|H h(x)-H h\left(x_{0}\right)\right| \\
& =\mid \operatorname{opt}_{y \in D}\left\{u(x, y)+\operatorname{opt}\left\{v(x, y), p_{i}(x, y)+q_{i}(x, y)\right.\right. \\
& \left.\left.\times h\left(a_{i}(x, y)\right): i=1,2,3\right\}\right\} \\
& \text { - opt }\left\{u\left(x_{0}, y\right)\right. \\
& + \text { opt }\left\{v\left(x_{0}, y\right), p_{i}\left(x_{0}, y\right)+q_{i}\left(x_{0}, y\right)\right. \\
& \left.\left.\times h\left(a_{i}\left(x_{0}, y\right)\right): i=1,2,3\right\}\right\} \\
& \leq \sup _{y \in D}\left\{\left|u(x, y)-u\left(x_{0}, y\right)\right|\right. \\
& +\max \left\{\left|v(x, y)-v\left(x_{0}, y\right)\right|,\right.
\end{aligned}
$$




$$
\begin{gathered}
\left|p_{i}(x, y)-p_{i}\left(x_{0}, y\right)\right| \\
+\mid q_{i}(x, y) h\left(a_{i}(x, y)\right)-q_{i}\left(x_{0}, y\right) \\
\left.\left.\times h\left(a_{i}\left(x_{0}, y\right)\right) \mid: i=1,2,3\right\}\right\} \\
\leq \sup _{y \in D}\left\{\left|u(x, y)-u\left(x_{0}, y\right)\right|\right. \\
+\max \left\{\left|v(x, y)-v\left(x_{0}, y\right)\right|,\left|p_{i}(x, y)-p_{i}\left(x_{0}, y\right)\right|\right. \\
+\left|q_{i}(x, y)-q_{i}\left(x_{0}, y\right)\right|\left|h\left(a_{i}(x, y)\right)\right| \\
+\left|q_{i}\left(x_{0}, y\right)\right|\left|h\left(a_{i}(x, y)\right)-h\left(a_{i}\left(x_{0}, y\right)\right)\right| \\
: i=1,2,3\}\} \\
\leq \frac{\epsilon}{4}+\max \left\{\frac{\epsilon}{4}, \frac{\epsilon}{4}+\frac{\epsilon}{4 M} M+\alpha \frac{\epsilon}{4}\right\} \\
<\epsilon, \quad
\end{gathered}
$$

which implies that $H h$ is continuous at $x_{0}$. Thus, $H$ is a selfmapping on $B C(S)$.

Given $\epsilon>0, x \in S$ and $h, g \in B C(S)$. Suppose that opt $_{y \in D}=\sup _{y \in D}$. Then, $\exists y, z \in D$ such that

$$
\begin{aligned}
& H h(x)<u(x, y)+\operatorname{opt}\{ v(x, y), p_{i}(x, y)+q_{i}(x, y) \\
& \times\left.h\left(a_{i}(x, y)\right): i=1,2,3\right\}+\epsilon, \\
& H g(x)<u(x, z)+\operatorname{opt}\{ v(x, z), p_{i}(x, z)+q_{i}(x, z) \\
& \times\left.g\left(a_{i}(x, z)\right): i=1,2,3\right\}+\epsilon, \\
& H h(x) \geq u(x, z)+\operatorname{opt}\left\{v(x, z), p_{i}(x, z)+q_{i}(x, z)\right. \\
&\left.\times h\left(a_{i}(x, z)\right): i=1,2,3\right\}, \\
& H g(x) \geq u(x, y)+\operatorname{opt}\left\{v(x, y), p_{i}(x, y)+q_{i}(x, y)\right. \\
&\left.\times g\left(a_{i}(x, y)\right): i=1,2,3\right\} .
\end{aligned}
$$

Using (20), we arrive at

$$
\begin{aligned}
& |H h(x)-H g(x)| \\
& <\max \left\{\operatorname { m a x } \left\{|| q_{i}(x, y) \mid\right.\right. \\
& \left.\quad \times\left|h\left(a_{i}(x, y)\right)-g\left(a_{i}(x, y)\right)\right|: i=1,2,3\right\}, \\
& \quad \max \left\{\left|q_{i}(x, z)\right|\right. \\
& \left.\left.\quad \times\left|h\left(a_{i}(x, z)\right)-g\left(a_{i}(x, z)\right)\right|: i=1,2,3\right\}\right\} \\
& +\epsilon \quad \max \left\{\left|q_{i}(x, y)\right|,\left|q_{i}(x, z)\right|\right\}\|h-g\|_{1}+\epsilon \\
& \leq \alpha\|h-g\|_{1}+\epsilon
\end{aligned}
$$

which implies that

$$
\|H h-H g\|_{1} \leq \varphi\left(\|h-g\|_{1}\right)+\epsilon, \quad \forall h, g \in B C(S),
$$

where $\varphi(\lambda)=\alpha \lambda, \lambda \in R^{+}$. Letting $\epsilon \rightarrow 0^{+}$, we get

$$
\|H h-H g\|_{1} \leq \varphi\left(\|h-g\|_{1}\right), \quad \forall h, g \in B C(S) .
$$

Similarly, we conclude that (23) holds for opt ${ }_{y \in D}=\inf _{y \in D}$.

Theorem 1 ensures that $H$ has a unique fixed point $w \in$ $B C(S)$ and $\left\{H^{n} h\right\}_{n \geq 1}$ converges to $w$ for each $h \in B C(S)$. It is obvious that $w$ is also a unique solution of the functional equation (1) in $B C(S)$. This completes the proof.

Dropping the compactness of $S$ and $\left(C_{2}\right)$ in the proof of Theorem 4 , we get the following result.

Theorem 5. Let $u, v, p_{i}, q_{i}: S \times D \rightarrow \mathbb{R}$ and $a_{i}: S \times D \rightarrow S$ for $i=1,2,3$ and satisfy conditions $\left(C_{1}\right)$ and $\left(C_{3}\right)$. Then, the functional equation (1) possesses a unique solution $w \in B(S)$ and $\left\{H^{n} h\right\}_{n \geq 1}$ converges to $w$ for each $h \in B(S)$, where $H$ is defined by (15).

Remark 6. If $u(x, y)=p_{i}(x, y)=0$, for $i=1,2,3$ and for all $(x, y) \in S \times D$, then Theorems 4 and 5 reduce to the results of Jiang et al. [8]. The example below shows that Theorems 4 and 5 extend substantially the results in [8].

Example 7. Let $X=Y=\mathbb{R}, S=[1,3], D=R^{+}$; then, Theorem 5 ensures that the following functional equation:

$$
\begin{aligned}
& f(x) \\
& =\underset{y \in D}{\operatorname{opt}}\left\{\left(1+\frac{x}{x+y^{2}}\right)\right. \\
& + \text { opt }\left\{\frac{x^{2}}{x+y+7}, \log \left(x+y^{2}+1\right)\right. \\
& +\sin (x+2 y) f\left(\frac{3 x^{2}+y^{3}}{x^{2}+y^{3}}\right), \\
& \sin \left(x+y^{2}+3\right) \\
& +\sin ^{2}(x y) f\left(\frac{5 x+y^{2}}{x+y^{2}}\right), \frac{x^{3}}{x+y^{2}+1} \\
& \left.\left.+\frac{x+\log \left(2 x y^{2}\right)}{3 x+\log \left(2 x y^{2}\right)} f\left(\frac{2 x^{2}+y}{x^{2}+y}\right)\right\}\right\} \text {, }
\end{aligned}
$$$$
\forall x \in S
$$

possesses a unique solution in $B(S)$. However, the corresponding results in $[8]$ are not applicable for the functional equation (24) because

$$
\left(1+\frac{x}{x+y^{2}}\right)>0, \quad \forall(x, y) \in S \times D .
$$

We point out that the functional equation (24) possesses also a unique solution in $B C(S)$. 

$B B(S)$.

In our next results, we discuss properties of solutions in

Theorem 8. Let $u, v, p_{i}, q_{i}: S \times D \rightarrow \mathbb{R}$ and $a_{i}: S \times D \rightarrow S$ for $i=1,2,3$ and satisfy the following conditions:

$\left(B_{1}\right) u, v$, and $p_{i}$ are bounded on $\bar{B}(0, k) \times D$ for $k \geq 1$ and $i=1,2,3$,

$\left(B_{2}\right) \max \left\{\left\|a_{i}(x, y)\right\|: i=1,2,3\right\} \leq\|x\|$, for $(x, y) \in S \times D$,

$\left(B_{3}\right)$ there exists a constant $\alpha$ such that $\sup _{(x, y) \in S \times D}\left\{\left|q_{i}(x, y)\right|: i=1,2,3\right\} \leq \alpha<1$.

Then, the functional equation (1) possesses a unique solution $w \in B B(S)$ and $\left\{H^{n} h\right\}_{n \geq 1}$ converges to $w$ for each $h \in B B(S)$, where $H$ is defined by (15).

Proof. It follows from $\left(B_{1}\right)$ and $\left(B_{2}\right)$ that for each $k \geq 1$ and $h \in B B(S)$, there exists $\beta(k)>0$ and $\eta(k, h)>0$ such that

$$
\begin{aligned}
& \sup _{(x, y) \in \bar{B}(0, k) \times D}\left\{|u(x, y)|,|v(x, y)|,\left|p_{i}(x, y)\right|: i=1,2,3\right\} \\
& \quad \leq \beta(k), \\
& \quad \sup _{(x, y) \in \bar{B}(0, k) \times D}\left\{\left|h\left(a_{i}(x, y)\right)\right|: i=1,2,3\right\} \leq \eta(k, h) .
\end{aligned}
$$

By virtue of $\left(B_{3}\right),(26)$, we know that

$$
\begin{aligned}
&|H h(x)| \leq \sup _{y \in D}\{|u(x, y)| \\
&+\max \left\{|v(x, y)|,\left|p_{i}(x, y)\right|+\left|q_{i}(x, y)\right|\right. \\
&\left.\left.\quad \times\left|h\left(a_{i}(x, y)\right)\right|: i=1,2,3\right\}\right\} \\
& \leq \beta(k)+\max \{\beta(k), \beta(k)+\eta(k, h)\} \\
& \leq 2 \beta(k)+\eta(k, h) .
\end{aligned}
$$

Thus, $H h$ is bounded; that is, $H$ is self-mapping on $B B(S)$.

Let $k \in N, x \in \bar{B}(0, k), g, h \in B B(S)$ and $\epsilon>0$. Suppose that opt $\mathrm{t}_{y \in D}=\sup _{y \in D}$. Then, there exists $y, z \in D$ satisfying

$$
\begin{aligned}
& H h(x)<u(x, y)+\operatorname{opt}\left\{v(x, y), p_{i}(x, y)+q_{i}(x, y)\right. \\
& \times\left.h\left(a_{i}(x, y)\right): i=1,2,3\right\}+\epsilon, \\
& H g(x)<u(x, z)+\operatorname{opt}\{\left\{(x, z), p_{i}(x, z)+q_{i}(x, z)\right. \\
&\left.\times g\left(a_{i}(x, z)\right): i=1,2,3\right\}+\epsilon, \\
& H h(x) \geq u(x, z)+\operatorname{opt}\left\{v(x, z), p_{i}(x, z)+q_{i}(x, z)\right. \\
&\left.\times h\left(a_{i}(x, z)\right): i=1,2,3\right\}, \\
& H g(x) \geq u(x, y)+\operatorname{opt}\left\{v(x, y), p_{i}(x, y)+q_{i}(x, y)\right. \\
&\left.\times g\left(a_{i}(x, y)\right): i=1,2,3\right\} .
\end{aligned}
$$

In terms of the above, $\left(B_{3}\right)$ and Lemma 2 , we get

$$
\begin{array}{r}
|H h(x)-H g(x)| \\
<\max \left\{\operatorname { m a x } \left\{\left|q_{i}(x, y)\right|\left|h\left(a_{i}(x, y)\right)-g\left(a_{i}(x, y)\right)\right|\right.\right. \\
: i=1,2,3\}, \\
\max \left\{\left|q_{i}(x, z)\right|\left|h\left(a_{i}(x, z)\right)-g\left(a_{i}(x, z)\right)\right|\right. \\
: i=1,2,3\}\}+\epsilon \\
\leq \alpha \max \left\{\left\{\left|h\left(a_{i}(x, y)\right)-g\left(a_{i}(x, y)\right)\right|: i=1,2,3\right\},\right. \\
\left.\quad\left\{\left|h\left(a_{i}(x, z)\right)-g\left(a_{i}(x, z)\right)\right|: i=1,2,3\right\}\right\} \\
+\epsilon, \quad
\end{array}
$$

which implies that

$$
|H h(x)-H g(x)| \leq \alpha d_{k}(h, g)+\epsilon, \quad \forall h, g \in B B(S) .
$$

In a similar way, we can show that (30) holds for opt $\mathrm{y}_{y \in D}=$ $\inf _{y \in D}$. It follows that

$$
d_{k}(H g, H h) \leq \varphi\left(d_{k}(h, g)\right)+\epsilon,
$$

where $\varphi(\lambda)=\alpha \lambda$ for $\lambda \in R^{+}$. As $\epsilon \rightarrow 0^{+}$in (31), we get that

$$
d_{k}(H g, H h) \leq \varphi\left(d_{k}(h, g)\right) .
$$

It follows from Theorem 2.2 in [3] that $H$ has a unique fixed point $w \in B B(S)$ and $\left\{H^{n} h\right\}_{n \geq 1}$ converges to $w$ for each $h \in$ $B B(S)$. Obviously, $w$ is also a unique solution of the functional equation (1). This completes the proof.

Remark 9. (1) If $u=v=q_{1}=q_{3}=p_{1}=p_{2}=0$, opt $t_{y \in D}=$ $\inf _{y \in D}$, and opt $=\max$, then Theorem 8 reduces to Theorem 3.4 of Bhakta and Choudhury [3] and a result of Bellman [1].

(2) If $v=p_{3}=q_{3}=0$, then Theorem 8 reduces to a result of Liu et al. [5].

(3) If we replace $u(x, y)$ by $p(x, y)+q(x, y) f(a(x, y))$ and $p_{1}=p_{2}=p_{3}=q_{3}=0$, then Theorem 8 reduces to Theorem 4.1 of Liu et al. [6], which, in turn, generalizes the results in $[1,3]$.

(4) Theorem 3.3 of Jiang et al. [8] is a particular case of Theorem 8.

(5) Theorem 4.1 of Pathak and Deepmala [10] is reduced to Theorem 8 , whenever $u=0$.

Theorem 10. Let $u, v, p_{i}, q_{i}: S \times D \rightarrow \mathbb{R}$ and $a_{i}: S \times D \rightarrow S$ for $i=1,2,3$, and let $(\varphi, \psi)$ be in $\left(\Phi_{2}\right)$ satisfying the following conditions:

$\left(B_{4}\right) \sup _{y \in D}\left\{|u(x, y)|,|v(x, y)|,\left|p_{i}(x, y)\right|: i=1,2,3\right\} \leq$ $\psi(\|x\|)$, for all $(x, y) \in S \times D$.

$\left(B_{5}\right) \max \left\{\left\|a_{i}(x, y)\right\|: i=1,2,3\right\} \leq \varphi(\|x\|)$, for all $(x, y) \in$ $S \times D$.

$\left(B_{6}\right) \max \left\{\left|q_{i}(x, y)\right|: i=1,2,3\right\} \leq 1$, for all $(x, y) \in S \times D$.

Then, the functional equation (1) possesses a solution $w \in$ $\mathrm{BB}(\mathrm{S})$ that satisfies the following conditions: 
$\left(B_{7}\right)$ the sequence $\left\{w_{n}\right\}_{n \geq 0}$ is defined by

$$
\begin{aligned}
& w_{0}(x)=\operatorname{opt}_{y \in D}\left\{u(x, y)+\operatorname{opt}\left\{v(x, y), p_{i}(x, y):\right.\right. \\
& i=1,2,3\}\}, \text { for all } x \in S ; \\
& w_{n}(x)=\operatorname{opt}_{y \in D}\left\{u ( x , y ) + \text { opt } \left\{v(x, y), p_{i}(x, y)+\right.\right. \\
& \left.\left.q_{i}(x, y) w_{n-1}\left(a_{i}(x, y)\right): i=1,2,3\right\}\right\}, \text { for all } x \in \\
& S, n \geq 1 \text { converges to } w .
\end{aligned}
$$

$\left(B_{8}\right) \lim _{n \rightarrow \infty} w\left(x_{n}\right)=0$ for any $x_{0} \in S,\left\{y_{n}\right\}_{n \geq 1} \subset D$ and $x_{n} \in\left\{a_{i}\left(x_{n-1}, y_{n-1}\right): i=1,2,3\right\}$, for all $n \in N$.

$\left(B_{9}\right) w$ is unique with respect to condition $\left(B_{8}\right)$.

Proof. Since $(\varphi, \psi)$ is in $\left(\Phi_{2}\right)$, it is easy to verify that

$$
\varphi(t)<t \text { for } t<0 .
$$

First of all we assert that the mapping $H$ defined by (15) is nonexpansive on $B B(S)$ on account of (33) and $\left(B_{5}\right)$; we obtain that

$$
\begin{array}{r}
\max \left\{\left\|a_{i}(x, y)\right\|: i=1,2,3\right\} \leq \varphi(\|x\|)<k, \\
\text { for }(x, y) \in \bar{B}(0, k) \times D,
\end{array}
$$

which implies that $\exists$ a constant $\theta(k, h)>0$ with

$$
\begin{array}{r}
\max \left\{\left|h\left(a_{i}(x, y)\right)\right|: i=1,2,3\right\} \leq \theta(k, h), \\
\text { for }(x, y) \in \bar{B}(0, k) \times D .
\end{array}
$$

By virtue of $\left(B_{4}\right),\left(B_{6}\right),(15),(35)$, and Lemmas 2 and 3, we deduce that

$$
\begin{aligned}
& |H h(x)|=\sup _{y \in D}\{|u(x, y)| \\
& +\mid \operatorname{opt}\left\{v(x, y), p_{i}(x, y)+q_{i}(x, y)\right. \\
& \left.\left.\quad \times h\left(a_{i}(x, y)\right)\right\} \mid: i=1,2,3\right\} \\
& \leq \sup _{y \in D}\{|u(x, y)|+\max \{|v(x, y)|, \\
& \qquad \quad\left|p_{i}(x, y)\right|+\left|q_{i}(x, y)\right| \\
& \left.\left.\leq \psi\left|\left(a_{i}(x, y)\right)\right|: i=1,2,3\right\}\right\} \\
& \leq 2 \psi(k)+\theta(k, h) \quad \text { for } x \in \bar{B}(0, k) .
\end{aligned}
$$

Thus, $H$ is self-mapping on $B B(S)$.

As in the proof of Theorem 8 , by $\left(B_{6}\right)$ we immediately conclude that for $h, g \in B B(S)$ and $k \geq 1$,

$$
d_{k}(H h, H g) \leq d_{k}(h, g),
$$

which yields that

$$
\begin{aligned}
d(H h, H g) & =\sum_{k=1}^{\infty} \frac{1}{2^{k}} \frac{d_{k}(H h, H g)}{1+d_{k}(H h, H g)} \\
& \leq \sum_{k=1}^{\infty} \frac{1}{2^{k}} \frac{d_{k}(h, g)}{1+d_{k}(h, g)}=d(h, g)
\end{aligned}
$$

$$
\text { Now we assert that for each } n \geq 0 \text {, }
$$

$$
\left|w_{n}(x)\right| \leq 2 \sum_{j=0}^{n} \psi\left(\varphi^{j}(\|x\|)\right), \quad x \in S .
$$

Now by $\left(B_{4}\right)$ we see that

$$
\begin{aligned}
&\left|w_{0}(x)\right| \leq \sup _{y \in D}\{|u(x, y)| \\
&\left.\quad+\max \left\{|v(x, y)|,\left|p_{i}(x, y)\right|: i=1,2,3\right\}\right\} \\
& \leq 2 \psi(\|x\|), \quad x \in S .
\end{aligned}
$$

That is, (39) is true for $n=0$. Suppose that (39) holds for some $n \geq 0$. From $\left(B_{4}\right)-\left(B_{6}\right)$ we know that

$$
\begin{aligned}
& \left|w_{n+1}(x)\right| \\
& =\mid \operatorname{opt}_{y \in D}\left\{u(x, y)+\operatorname{opt}\left\{v(x, y), p_{i}(x, y)+q_{i}(x, y)\right.\right. \\
& \left.\left.\times w_{n}\left(a_{i}(x, y)\right): i=1,2,3\right\}\right\} \\
& \leq \sup _{y \in D}\{|u(x, y)| \\
& +\max \left\{|v(x, y)|,\left|p_{i}(x, y)\right|+\left|q_{i}(x, y)\right|\right. \\
& \left.\left.\times\left|w_{n}\left(a_{i}(x, y)\right)\right|: i=1,2,3\right\}\right\} \\
& \leq \sup _{y \in D}\{2 \psi(\|x\|) \\
& \left.+\max \left\{2 \sum_{j=0}^{n} \psi\left(\varphi^{j}\left(\left\|a_{i}(x, y)\right\|\right)\right): i=1,2,3\right\}\right\} \\
& \leq 2 \sum_{j=0}^{n+1} \psi\left(\varphi^{j}(\|x\|)\right) \text {. }
\end{aligned}
$$

Hence, (39) holds for $n \geq 0$.

Next we claim that $\left\{w_{n}\right\}_{n \geq 0}$ is a cauchy sequence in $B B(S)$. Given $k \geq 1$ and $x_{0} \in \bar{B}(0, k)$. Let $\epsilon>0, n, m \in N$. Suppose that opt $\mathrm{t}_{y \in D}=\sup _{y \in D}$. Then, we select $y, z \in D$ such that

$$
\begin{aligned}
w_{n}\left(x_{0}\right)< & u\left(x_{0}, y\right)+\text { opt }\{ \\
& \left(x_{0}, y\right), p_{i}\left(x_{0}, y\right)+q_{i}\left(x_{0}, y\right) \\
& \left.\times w_{n-1}\left(a_{i}\left(x_{0}, y\right)\right): i=1,2,3\right\} \\
+ & \frac{\epsilon}{2},
\end{aligned}
$$




$$
\begin{gathered}
w_{n+m}\left(x_{0}\right)<\begin{array}{l}
u\left(x_{0}, z\right) \\
+\operatorname{opt}\left\{v\left(x_{0}, z\right), p_{i}\left(x_{0}, z\right)+q_{i}\left(x_{0}, z\right)\right. \\
\left.\quad \times w_{n+m-1}\left(a_{i}\left(x_{0}, z\right)\right): i=1,2,3\right\}
\end{array} \\
\left.+\frac{\epsilon}{2}, \quad \times w_{n-1}\left(a_{i}\left(x_{0}, z\right)\right): i=1,2,3\right\}, \\
w_{n}\left(x_{0}\right) \geq u\left(x_{0}, z\right)+\operatorname{opt}\left\{v\left(x_{0}, z\right), p_{i}\left(x_{0}, z\right)+q_{i}\left(x_{0}, z\right)\right. \\
w_{n+m}\left(x_{0}\right) \geq u\left(x_{0}, y\right) \quad \operatorname{opt}\left\{v\left(x_{0}, y\right), p_{i}\left(x_{0}, y\right)+q_{i}\left(x_{0}, y\right)\right. \\
\left.\quad \times w_{n+m-1}\left(a_{i}\left(x_{0}, y\right)\right): i=1,2,3\right\} .
\end{gathered}
$$

In view of $(42),\left(B_{6}\right)$, and Lemma 2 , we infer that

$$
\begin{aligned}
& w_{n+m}\left(x_{0}\right)-w_{n}\left(x_{0}\right) \\
& \leq \max \left\{\left|q_{i}\left(x_{0}, z\right)\right|\left|w_{n+m-1}\left(a_{i}\left(x_{0}, z\right)\right)-w_{n-1}\left(a_{i}\left(x_{0}, z\right)\right)\right|\right. \\
& : i=1,2,3\}+\frac{\epsilon}{2} \\
& \leq \max \left\{\left|q_{i}\left(x_{0}, z\right)\right|: i=1,2,3\right\} \\
& \quad \times \max \left\{\left|w_{n+m-1}\left(a_{i}\left(x_{0}, z\right)\right)-w_{n-1}\left(a_{i}\left(x_{0}, z\right)\right)\right|\right. \\
& \quad: i=1,2,3\}+\frac{\epsilon}{2} \\
& \leq \max \left\{\left|w_{n+m-1}\left(a_{i}\left(x_{0}, z\right)\right)-w_{n-1}\left(a_{i}\left(x_{0}, z\right)\right)\right|\right. \\
& : i=1,2,3\}+\frac{\epsilon}{2}, \\
& w_{n+m}\left(x_{0}\right)-w_{n}\left(x_{0}\right) \\
& \geq-\max \left\{\left|w_{n+m-1}\left(a_{i}\left(x_{0}, y\right)\right)-w_{n-1}\left(a_{i}\left(x_{0}, y\right)\right)\right|\right. \\
& : i=1,2,3\}-\frac{\epsilon}{2},
\end{aligned}
$$

which means that

$$
\begin{gathered}
w_{n+m}\left(x_{0}\right)-w_{n}\left(x_{0}\right) \\
<\max \left\{\operatorname { m a x } \left\{\left|w_{n+m-1}\left(a_{i}\left(x_{0}, z\right)\right)-w_{n-1}\left(a_{i}\left(x_{0}, z\right)\right)\right|\right.\right. \\
: i=1,2,3\}, \\
\max \left\{\left|w_{n+m-1}\left(a_{i}\left(x_{0}, y\right)\right)-w_{n-1}\left(a_{i}\left(x_{0}, y\right)\right)\right|\right. \\
: i=1,2,3\}\}+\frac{\epsilon}{2} \\
=\left|w_{n+m-1}\left(x_{1}\right)-w_{n-1}\left(x_{1}\right)\right|+\frac{\epsilon}{2},
\end{gathered}
$$

for some $y_{1} \in\{y, z\}$ and $x_{1} \in\left\{a_{i}\left(x_{0}, y_{1}\right): i=1,2,3\right\}$.
Similarly, we conclude that the above inequality (44) holds for opt $\mathrm{t}_{y \in D}=\inf _{y \in D}$. Proceeding in this way, we select $y_{j} \in D$ and $x_{j} \in\left\{a_{i}\left(x_{j-1}, y_{j}\right): i=1,2,3\right\}$ for $j=2,3, \ldots, n$ such that

$$
\begin{gathered}
\left|w_{n+m-1}\left(x_{1}\right)-w_{n-1}\left(x_{1}\right)\right|<\left|w_{n+m-2}\left(x_{2}\right)-w_{n-2}\left(x_{2}\right)\right|+2^{-2} \epsilon, \\
\left|w_{n+m-2}\left(x_{2}\right)-w_{n-2}\left(x_{2}\right)\right|<\left|w_{n+m-3}\left(x_{3}\right)-w_{n-3}\left(x_{3}\right)\right|+2^{-3} \epsilon, \\
\vdots \\
\left|w_{m+1}\left(x_{n-1}\right)-w_{1}\left(x_{n-1}\right)\right|<\left|w_{m}\left(x_{n}\right)-w_{0}\left(x_{n}\right)\right|+2^{-n} \epsilon .
\end{gathered}
$$

It follows from $\left(B_{5}\right),(33),(39),(44)$, and (45) that

$$
\begin{aligned}
& \left|w_{n+m}\left(x_{0}\right)-w_{n}\left(x_{0}\right)\right| \\
& <\left|w_{m}\left(x_{n}\right)-w_{0}\left(x_{n}\right)\right|+\sum_{i=1}^{n} 2^{-i} \epsilon \\
& <\left|w_{m}\left(x_{n}\right)\right|+\left|w_{0}\left(x_{n}\right)\right|+\epsilon \\
& \leq 2 \sum_{i=0}^{m} \psi\left(\varphi^{i}\left(\left\|x_{n}\right\|\right)\right)+2 \psi\left(\left\|x_{n}\right\|\right)+\epsilon \\
& \leq 2 \sum_{i=0}^{m} \psi\left(\varphi^{i+n}\left(\left\|x_{0}\right\|\right)\right)+2 \psi\left(\varphi^{n}\left(\left\|x_{0}\right\|\right)\right)+\epsilon \\
& \leq 2 \sum_{j=n-1}^{\infty} \psi\left(\varphi^{j}(k)\right)+\epsilon,
\end{aligned}
$$

which implies that

$$
d_{k}\left(w_{n+m}, w_{n}\right) \leq 2 \sum_{j=n-1}^{\infty} \psi\left(\varphi^{j}(k)\right)+\epsilon .
$$

As $\epsilon \rightarrow 0^{+}$in the above inequality, we deduce that

$$
d_{k}\left(w_{n+m}, w_{n}\right) \leq 2 \sum_{j=n-1}^{\infty} \psi\left(\varphi^{j}(k)\right),
$$

which yields that $\left\{w_{n}\right\}_{n \geq 0}$ is a cauchy sequence in $(B B(S), d)$ since $\sum_{i=0}^{\infty} \psi\left(\varphi^{n}(t)\right)<\infty$, for each $t>0$. Suppose that $\left\{w_{n}\right\}_{n \geq 0}$ converges to some $w \in B B(S)$. Notice that $H$ is nonexpansive. It follows that

$$
\begin{aligned}
d(w, H w) & \leq d\left(w, H w_{n}\right)+d\left(H w_{n}, H w\right) \\
& \leq d\left(w, w_{n+1}\right)+d\left(w_{n}, w\right) \\
& \longrightarrow 0 \text { as } n \longrightarrow \infty .
\end{aligned}
$$

That is, $H w=w$. Thus, the functional equation (1) possess a solution $w$.

Now we show that $\left(B_{8}\right)$ holds. Let $\epsilon>0, x_{0} \in S,\left\{y_{n}\right\}_{n \geq 1} \subset$ $D$, and $x_{n} \in\left\{a_{i}\left(x_{n-1}, y_{n}\right), i=1,2,3\right\}$, for $n \geq 1$. Put $k=$ $\left[\left\|x_{0}\right\|\right]+1$. Then, there exists a positive integer $m$ satisfying

$$
d_{k}\left(w, w_{n}\right)+2 \sum_{j=n}^{\infty} \psi\left(\varphi^{j}(k)\right)<\epsilon, \quad \text { for } n>m .
$$


By (39), $\left(B_{5}\right)$, and (50), we infer that, for $n>m$,

$$
\begin{aligned}
\left|w\left(x_{n}\right)\right| & \leq\left|w\left(x_{n}\right)-w_{n}\left(x_{n}\right)\right|+\left|w_{n}\left(x_{n}\right)\right| \\
& \leq d_{k}\left(w, w_{n}\right)+2 \sum_{j=0}^{\infty} \psi\left(\varphi^{j}\left(\left\|x_{n}\right\|\right)\right) \\
& \leq d_{k}\left(w, w_{n}\right)+2 \sum_{j=n}^{\infty} \psi\left(\varphi^{j}(k)\right) \\
& \leq \epsilon,
\end{aligned}
$$

which means that $\lim _{n \rightarrow \infty} w\left(x_{n}\right)=0$.

Finally, we show that $\left(B_{9}\right)$ holds. Suppose that the functional equation (1) possesses another solution $h \in B B(S)$, which satisfies condition $\left(B_{8}\right)$. Let $\epsilon>0$ and $x_{0} \in S$. If opt $_{y \in D}=\sup _{y \in D}$, then there exists $y, z \in S$ such that

$$
\begin{aligned}
& w\left(x_{0}\right)<u\left(x_{0}, y\right)+\operatorname{opt}\left\{v\left(x_{0}, y\right), p_{i}\left(x_{0}, y\right)+q_{i}\left(x_{0}, y\right)\right. \\
& \times\left.w\left(a_{i}\left(x_{0}, y\right)\right): i=1,2,3\right\}+\frac{\epsilon}{2}, \\
& h\left(x_{0}\right)<u\left(x_{0}, z\right)+\operatorname{opt}\{ v\left(x_{0}, z\right), p_{i}\left(x_{0}, z\right)+q_{i}\left(x_{0}, z\right) \\
& \times\left.h\left(a_{i}\left(x_{0}, z\right)\right): i=1,2,3\right\}+\frac{\epsilon}{2}, \\
& w\left(x_{0}\right) \geq u\left(x_{0}, z\right)+\operatorname{opt}\{\left\{\left(x_{0}, z\right), p_{i}\left(x_{0}, z\right)+q_{i}\left(x_{0}, z\right)\right. \\
& \times\left.\times\left(a_{i}\left(x_{0}, z\right)\right): i=1,2,3\right\}, \\
& h\left(x_{0}\right) \geq u\left(x_{0}, y\right)+\text { opt }\left\{v\left(x_{0}, y\right), p_{i}\left(x_{0}, y\right)+q_{i}\left(x_{0}, y\right)\right. \\
&\left.\times h\left(a_{i}\left(x_{0}, y\right)\right): i=1,2,3\right\} .
\end{aligned}
$$

Using Lemma 2, $\left(B_{6}\right)$, and (52), we get

$$
\begin{aligned}
& w\left(x_{0}\right)-h\left(x_{0}\right) \\
& <\max \left\{\left|q_{i}\left(x_{0}, y\right)\right|\left|w\left(a_{i}\left(x_{0}, y\right)\right)-h\left(a_{i}\left(x_{0}, y\right)\right)\right|\right. \\
& : i=1,2,3\}+\frac{\epsilon}{2} \\
& \leq \max \left\{\left|q_{i}\left(x_{0}, y\right)\right|: i=1,2,3\right\} \\
& \quad \times \max \left\{\left|w\left(a_{i}\left(x_{0}, y\right)\right)-h\left(a_{i}\left(x_{0}, y\right)\right)\right|: i=1,2,3\right\}+\frac{\epsilon}{2} \\
& \leq \max \left\{\left|w\left(a_{i}\left(x_{0}, y\right)\right)-h\left(a_{i}\left(x_{0}, y\right)\right)\right|: i=1,2,3\right\}+\frac{\epsilon}{2}, \\
& w\left(x_{0}\right)-h\left(x_{0}\right) \\
& \geq-\max \left\{\left|w\left(a_{i}\left(x_{0}, z\right)\right)-h\left(a_{i}\left(x_{0}, z\right)\right)\right|: i=1,2,3\right\}-\frac{\epsilon}{2}
\end{aligned}
$$

which implies that

$$
\begin{aligned}
& \left|w\left(x_{0}\right)-h\left(x_{0}\right)\right| \\
& <\max \left\{\max \left\{\left|w\left(a_{i}\left(x_{0}, y\right)\right)-h\left(a_{i}\left(x_{0}, y\right)\right)\right|: i=1,2,3\right\},\right. \\
& \left.\quad \max \left\{\left|w\left(a_{i}\left(x_{0}, z\right)\right)-h\left(a_{i}\left(x_{0}, z\right)\right)\right|: i=1,2,3\right\}\right\} \\
& +\frac{\epsilon}{2} \\
& =\left|w\left(x_{1}\right)-h\left(x_{1}\right)\right|+\frac{\epsilon}{2}
\end{aligned}
$$

for some $y_{1} \in\{y, z\}$ and $x_{1} \in\left\{a_{i}\left(x_{0}, y_{i}\right): i=1,2,3\right\}$.

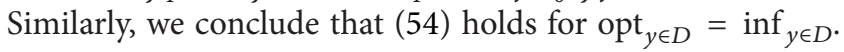
Proceeding in this way, we select $y_{j} \in D$ and $x_{j} \in$ $\left\{a_{i}\left(x_{j-1}, y_{j}\right)\right\}$ for $j=2,3, \ldots, n$ satisfying

$$
\begin{gathered}
\left|w\left(x_{1}\right)-h\left(x_{1}\right)\right|<\left|w\left(x_{2}\right)-h\left(x_{2}\right)\right|+2^{-2} \epsilon, \\
\left|w\left(x_{2}\right)-h\left(x_{2}\right)\right|<\left|w\left(x_{3}\right)-h\left(x_{3}\right)\right|+2^{-3} \epsilon, \\
\vdots \\
\left|w\left(x_{n-1}\right)-h\left(x_{n-1}\right)\right|<\left|w\left(x_{n}\right)-h\left(x_{n}\right)\right|+2^{-n} \epsilon .
\end{gathered}
$$

Combining (54) and (55), we obtain that

$$
\begin{aligned}
\left|w\left(x_{0}\right)-h\left(x_{0}\right)\right| & <\left|w\left(x_{n}\right)-h\left(x_{n}\right)\right|+\sum_{j=1}^{n} 2^{-j} \epsilon \\
& <\left|w\left(x_{n}\right)-h\left(x_{n}\right)\right|+\epsilon .
\end{aligned}
$$

Letting $n \rightarrow \infty$ in the above inequalities, by $\left(B_{8}\right)$ we get that

$$
\left|w\left(x_{0}\right)-h\left(x_{0}\right)\right| \leq \epsilon .
$$

As $\epsilon \rightarrow 0^{+}$in the above inequality, we know that $w\left(x_{0}\right)=$ $h\left(x_{0}\right)$. This completes the proof.

Remark 11. (1) Theorem 10 extends, improves, and unifies the results in Liu et al. $[5,6]$.

(2) In case $u=p_{1}=p_{2}=p_{3}=0$ and $(\varphi, \psi) \in \Phi_{3}$, then Theorem 10 reduces to Theorem 3.4 of Jiang et al. [8], which, in turn, generalizes and unifies the results of Bellman [1], Bhakta and Choudhury [3], Liu [11], and Liu and Ume [4].

(3) If we replace $p_{1}=p_{2}=p_{3}=0, q_{1}=q_{2}=q_{3}=$ $(1-\lambda), a_{1}=a_{2}=a_{3}=a(x, y), v(x, y)=(1-\lambda) v_{1}(x, y)$, $u(x, y)=\lambda[u(x, y)+f(a(x, y))], \psi(t)=M t$, and $\varphi \in \Phi_{4}$, for $(x, y) \in S \times D, t \in R^{+}$, where $\lambda$ is a constant in $[0,1]$ and $M$ is a positive constant, then Theorem 10 reduces to Theorem 3.1 of Liu and Ume [4], which, in turn, generalizes Theorem 3.5 in [3], Theorem 2.4 in [2], and a result in [1].

(4) Dropping condition $\left(B_{6}\right)$ and replacing opt ${ }_{y \in D}=$ inf $_{y \in D}$, opt $=\max , q_{1}=q_{2}=1, u=p_{1}=p_{3}=q_{3}=0$, then Theorem 10 reduces to Theorem 3.4 of Liu and Kang [7], which, in turn, generalizes the results in $[1,3,7]$.

The example below demonstrates that Theorem 10 generalizes, extends, and unifies the results in $[1-8,11]$. 
Example 12. Let $X=Y=R, S=D=[1, \infty), \psi(t)=4 t^{4}$, $\varphi(t)=t / 2$, for all $t \in R^{+}$. Consider the following functional equation:

$$
\begin{aligned}
& f(x) \\
& =\underset{y \in D}{\operatorname{opt}}\left\{x^{4}\left(1+\frac{1}{x+y^{2}}\right)\right. \\
& + \text { opt }\left\{x^{3}\left(1+\left|\sin ^{2}(x+2 y+3)\right|\right), \frac{2 x^{3}}{1+x+y}\right. \\
& +\sin ^{2}(x+2 y) f\left(\frac{x^{2}}{1+x^{2} y^{2}}\right), \\
& \frac{x^{8}}{x^{5}+\cos ^{2}(x+2 y-1)} \\
& +\frac{1}{1+x y} f\left(\frac{x^{3}}{1+x^{2}+y^{2}}\right), \frac{x^{4}}{1+x y} \\
& +\cos ^{2}\left(x+y^{3}\right) \\
& \left.\left.\times f\left(\frac{x}{2+x y^{2}} \sin ^{2}(x+2 y)\right)\right\}\right\}, \\
& \forall x \in S .
\end{aligned}
$$

It follows from Theorem 10 that the functional equation (58) possesses a solution $w \in B B(S)$. However, the corresponding results in $[1-8,11]$ are not valid for the functional equation (58) because

$$
\left|x^{4}\left(1+\frac{1}{x+y^{2}}\right)\right| \leq M|x|
$$

does not hold for $(x, y)=(1+M, 1) \in S \times D$, where $M$ is a positive constant; also

$$
x^{4}\left(1+\frac{1}{x+y^{2}}\right)>0, \quad x^{3}\left(1+\left|\sin ^{2}(x+2 y+3)\right|\right)>0 .
$$

\section{Conflict of Interests}

The author declares that there is no conflict of interests regarding the publication of this paper.

\section{Acknowledgments}

The author is grateful to the editor and the anonymous referees for their valuable comments and suggestions improving the exposition of the paper. The research of the author is supported by the Department of Science and Technology, Government of India, under INSPIRE Fellowship Program no. DST/INSPIRE Fellowship/2013/[182].

\section{References}

[1] R. Bellman, Dynamic Programming, Princeton University Press, Princeton, NJ, USA, 1957.

[2] P. C. Bhakta and S. Mitra, "Some existence theorems for functional equations arising in dynamic programming," Journal of Mathematical Analysis and Applications, vol. 98, no. 2, pp. 348-362, 1984.

[3] P. C. Bhakta and S. R. Choudhury, "Some existence theorems for functional equations arising in dynamic programming II," Journal of Mathematical Analysis and Applications, vol. 131, no. 1, pp. 217-231, 1988.

[4] Z. Liu and J. S. Ume, "On properties of solutions for a class of functional equations arising in dynamic programming," Journal of Optimization Theory and Applications, vol. 117, no. 3, pp. 533551, 2003.

[5] Z. Liu, R. P. Agarwal, and S. M. Kang, "On solvability of functional equations and system of functional equations arising in dynamic programming," Journal of Mathematical Analysis and Applications, vol. 297, no. 1, pp. 111-130, 2004.

[6] Z. Liu, Y. Xu, J. S. Ume, and S. M. Kang, "Solutions to two functional equations arising in dynamic programming," Journal of Computational and Applied Mathematics, vol. 192, no. 2, pp. 251-269, 2006

[7] Z.-Q. Liu and S. M. Kang, "Existence and uniqueness of solutions for two classes of functional equations arising in dynamic programming," Acta Mathematicae Applicatae Sinica. English Series, vol. 23, no. 2, pp. 195-208, 2007.

[8] G. Jiang, S. M. Kang, and Y. C. Kwun, "Solvability and algorithms for functional equations originating from dynamic programming," Fixed Point Theory and Applications, vol. 2011, Article ID 701519, 30 pages, 2011.

[9] D. W. Boyd and J. S. W. Wong, "On nonlinear contractions," Proceedings of the American Mathematical Society, vol. 20, pp. 458-464, 1969.

[10] H. K. Pathak and Deepmala, "Existence and uniqueness of solutions of functional equations arising in dynamic programming," Applied Mathematics and Computation, vol. 218, no. 13, pp. 72217230, 2012.

[11] Z. Liu, "Existence theorems of solutions for certain classes of functional equations arising in dynamic programming," Journal of Mathematical Analysis and Applications, vol. 262, no. 2, pp. 529-553, 2001.

[12] R. P. Agarwal, N. Hussain, and M.-A. Taoudi, "Fixed point theorems in ordered Banach spaces and applications to nonlinear integral equations," Abstract and Applied Analysis, vol. 2012, Article ID 245872, 15 pages, 2012.

[13] Deepmala and H. K. Pathak, "A study on some problems on existence of solutions for nonlinear functional-integral equations," Acta Mathematica Scientia B, vol. 33, no. 5, pp. 13051313, 2013.

[14] N. Hussain and M. A. Taoudi, "Krasnosel'skii-type fixed point theorems with applications to Volterra integral equations," Fixed Point Theory and Applications, vol. 2013, article 196, 2013.

[15] N. Hussain, A. R. Khan, and R. P. Agarwal, "Krasnosel'skii and Ky Fan type fixed point theorems in ordered Banach spaces," Journal of Nonlinear and Convex Analysis, vol. 11, no. 3, pp. 475489, 2010.

[16] Z. Liu, H. Dong, and S. M. Kang, "Solving a class of func- tional equations using fixed point theorems," Journal of Inequalities and Applications, vol. 2013, article 516, 2013. 


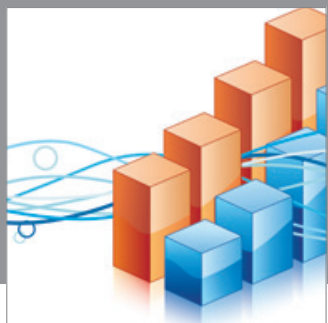

Advances in

Operations Research

mansans

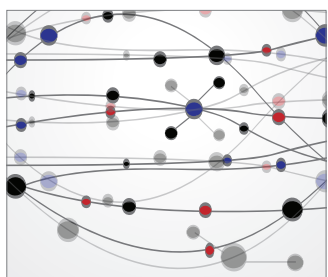

The Scientific World Journal
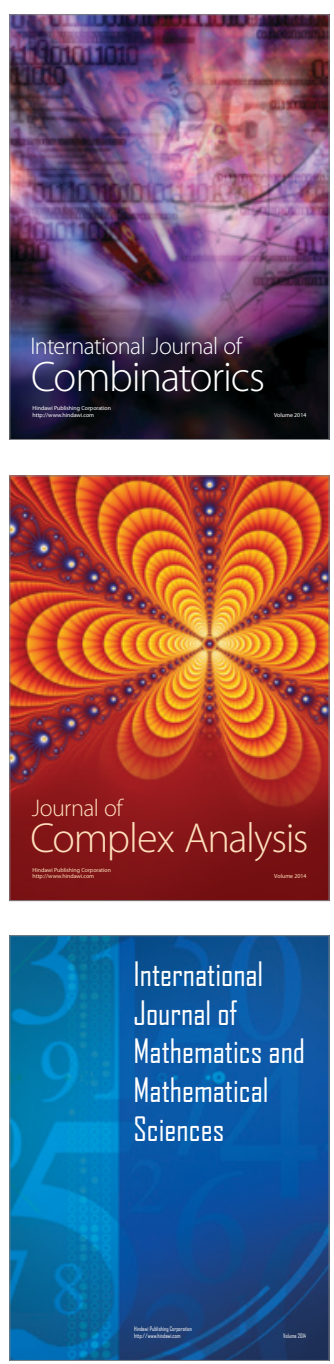
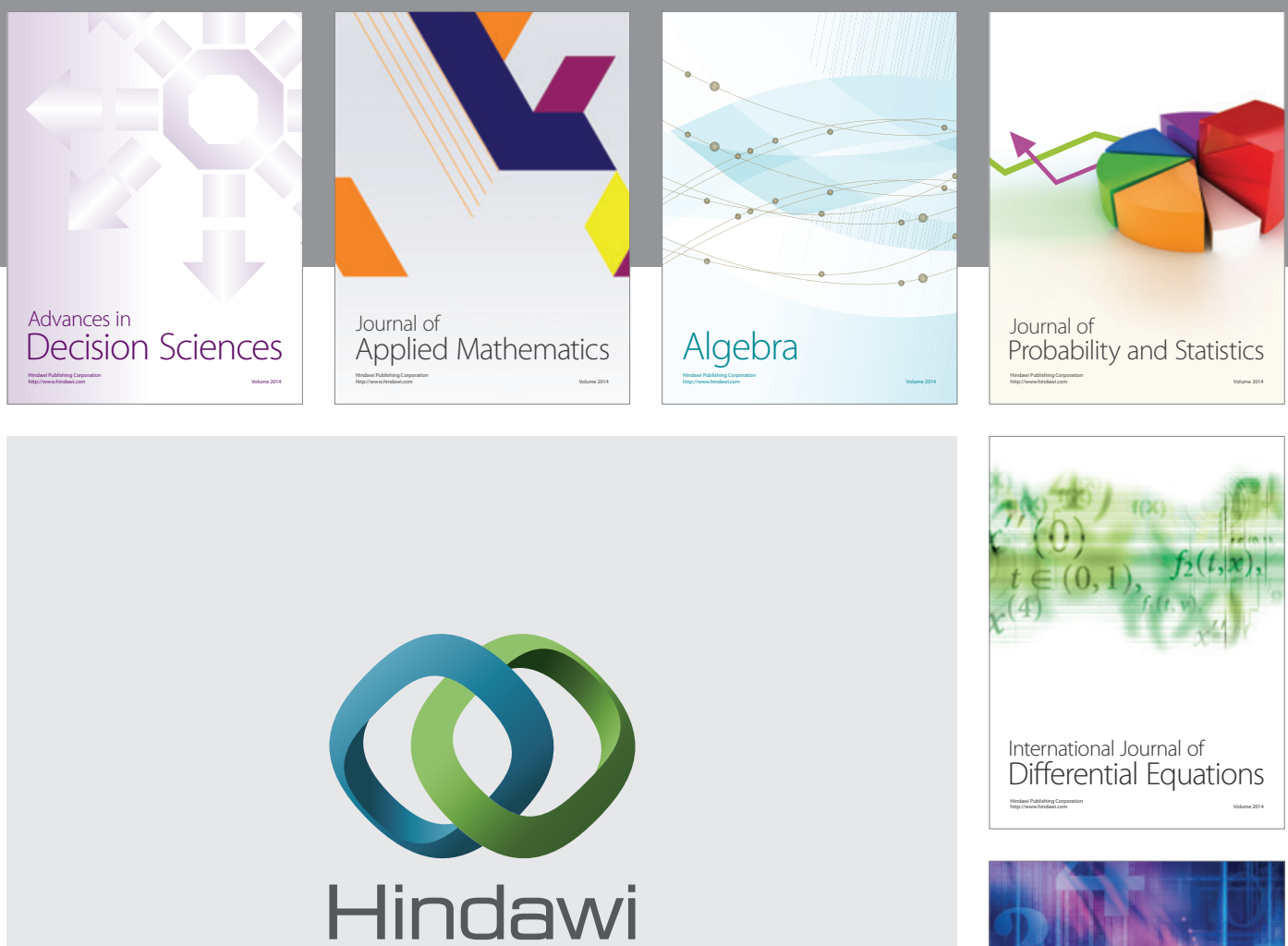

Submit your manuscripts at http://www.hindawi.com
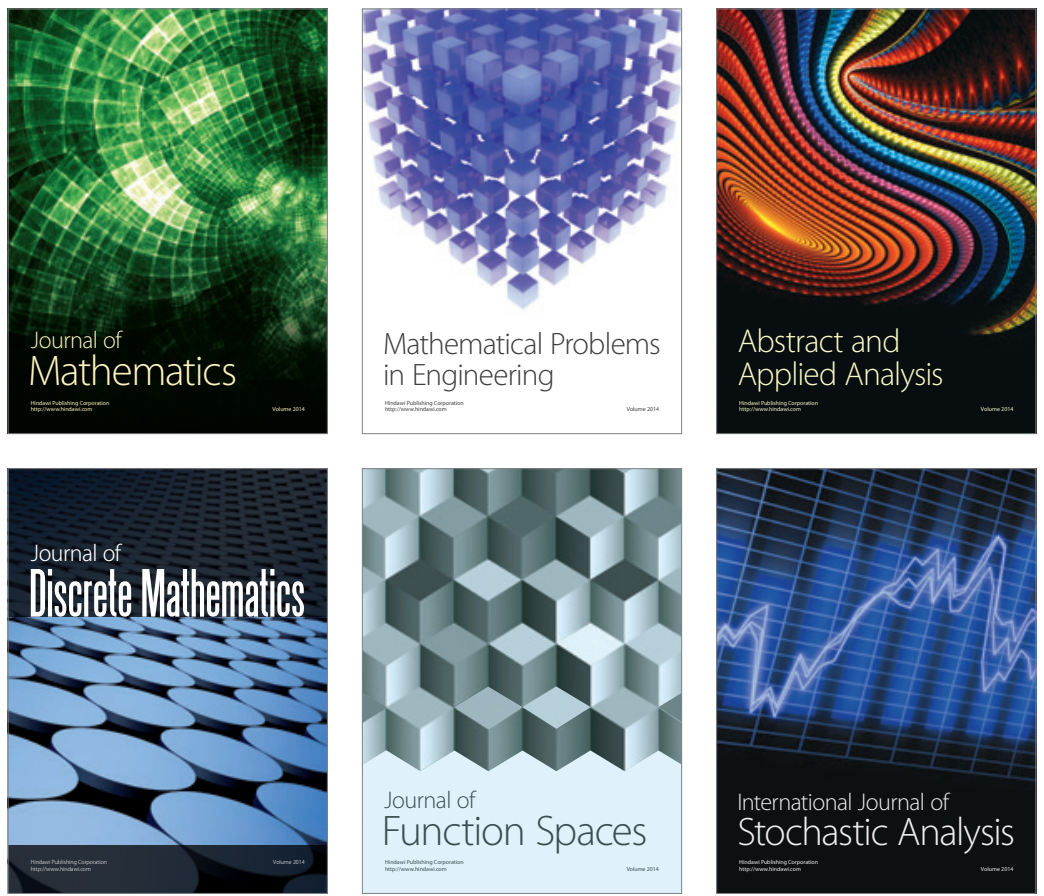

Journal of

Function Spaces

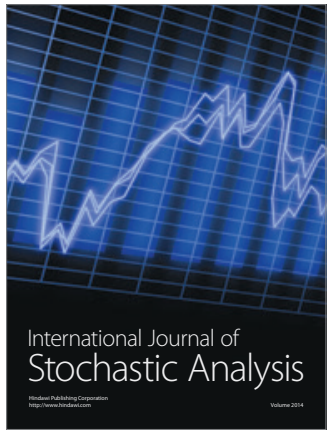

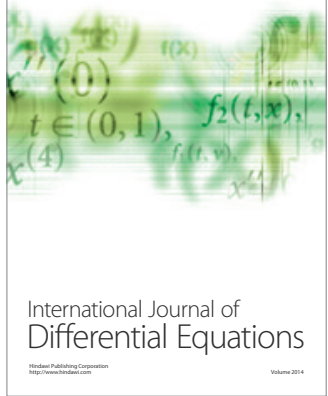
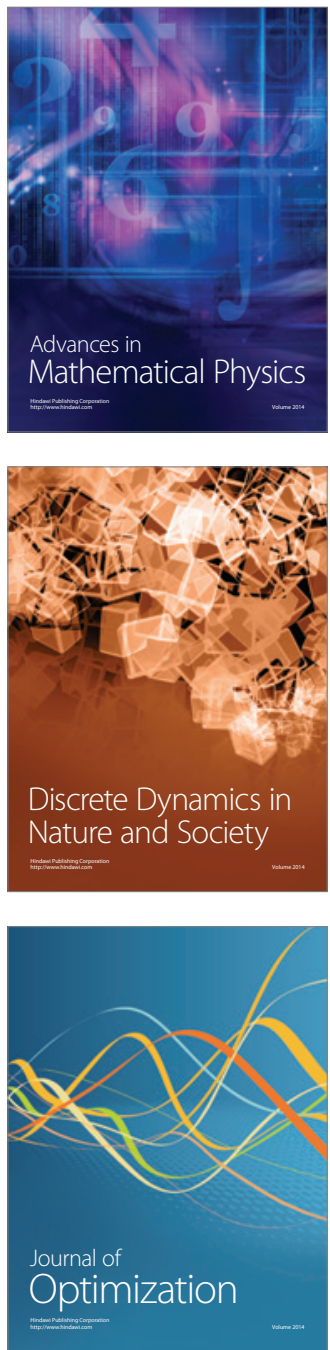